\title{
Isolation and X-ray Crystal Structure of an Electrogenerated TEMPO-N Charge-Transfer Complex
}

\author{
Hosea M. Nelson ${ }^{1 *}$, Juno C. Siu ${ }^{2}$, Ambarneil Saha ${ }^{1}$, Duilio Cascio ${ }^{1,3}$, Song-Bai Wu ${ }^{1,4}$, Chenxi \\ Lu $^{1}$, José A. Rodríguez ${ }^{1,3}$, K. N. Houk ${ }^{*}$, Song Lin ${ }^{2 *}$
}

${ }^{1}$ Department of Chemistry and Biochemistry, University of California, Los Angeles, Los Angeles California 90095, USA.

${ }^{2}$ Department of Chemistry and Chemical Biology, Cornell University, Ithaca, NY 14853, USA.

${ }^{3}$ UCLA-DOE Institute for Genomics \& Proteomics, University of California, Los Angeles, California 90095, USA.

${ }^{4}$ Shenzhen Grubbs Institute and Department of Chemistry, Southern University of Science and Technology, Shenzhen 518055, China.

*Correspondence to: hosea@chem.ucla.edu, houk@chem.ucla.edu, songlin@,cornell.edu

Abstract: Recent advances in radical-based catalytic reactions have created an increasing demand for the understanding of their mechanistic underpinnings. Structural elucidation of transient reactive intermediates via diffraction techniques, though rarely possible, is one of the most decisive ways to support such mechanistic hypotheses. Here we present the isolation, structural elucidation, and theoretical analysis of an electrochemically generated and catalytically relevant chargetransfer species formed between the azidyl radical and (2,2,6,6-tetramethylpiperidin-1-yl)oxyl (TEMPO). The unusual bent $\mathrm{N}-\mathrm{N}-\mathrm{N}$ angle and the pancake bonding between these two fragments highlight the weak bonding interactions present in this complex. This X-ray structure validates computational predictions as well as mechanistic proposals of TEMPO-mediated radical azidation reactions.

Charge-transfer complexes (CTCs) are non-covalent adducts comprised of an electron donor and an electron acceptor associated via weak molecular interactions arising from transfer of charge (1). These species have been postulated as key intermediates in many redox organic transformations, frequently mediating single-electron transfer events between an organic substrate and a redox agent. In particular, recent advances in radical chemistry feature the strategic exploitation of the unique characteristics of CTCs in contexts of photoredox catalysis $(2,3)$, electrocatalysis $(4,5)$, 
and biocatalysis $(6,7)$, enabling new modes of reactivity that were previously challenging to access. Despite their emerging role in radical catalysis, the short lifetimes of such CTCs have made their solid-state and solution-state structural determination difficult. Literature examples of CTC crystal structures (8-11) are most commonly reported for species that make use of polyaromatic precursors where $\pi$-stacking biases ordered packing. Such designer complexes have prominent applications in materials chemistry but are rarely relevant to catalysis (12). Here, we report a highresolution X-ray crystal structure of an anodically generated TEMPO-N 3 CTC, a metastable crystalline adduct that has been shown to be a crucial intermediate in the organocatalyzed electrochemical diazidation (5) and azidooxygenation of alkenes (4).

We first discovered the TEMPO- $\mathrm{N}_{3}$ CTC during our investigation of the electrochemical azidooxygenation of alkenes (Fig. 1) (4). Extensive spectroscopic and voltammetric studies strongly supported the formation of a TEMPO- $\mathrm{N}_{3}$ CTC (1) upon electrochemical generation of oxoammonium ion $\mathrm{TEMPO}^{+}$followed by its association with $\mathrm{N}_{3}{ }^{-}$. The formation of a reactive adduct of TEMPO and $\mathrm{N}_{3}$ has also been postulated in previous studies (13), but few structural characterization or reactivity studies of such an adduct were reported (14). Due to the instability of the CTC (half-life in MeCN solution $\sim 7 \mathrm{~min}$ ), structural insight relied on DFT calculations, which suggested four possible structures, with $\mathbf{1}$ being the lowest in energy and most consistent with spectroscopic data (Fig. 1) (4).
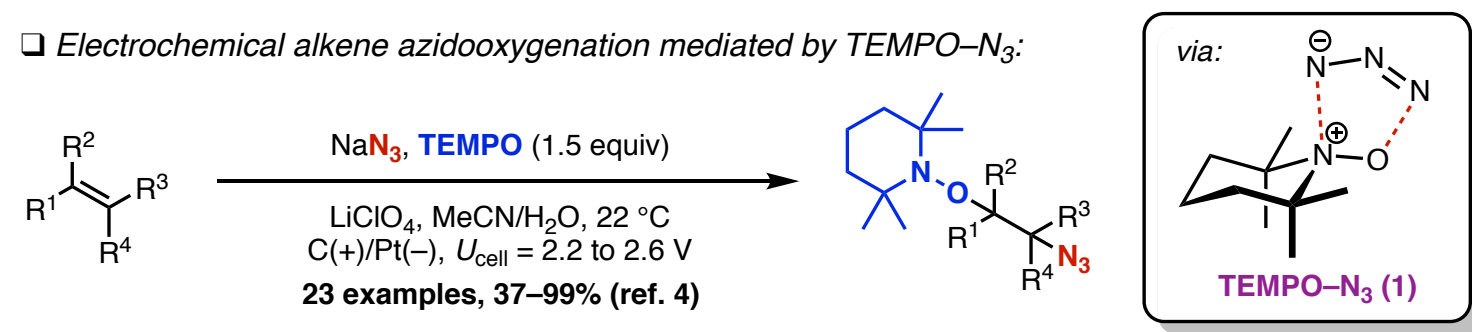

Fig. 1. Electrochemical alkene difunctionalization mediated by the TEMPO-N ${ }_{3}$ CTC (1).

To provide a more definitive structural confirmation, we attempted to collect diffraction data from the CTC using electron crystallography (15). Cryo-electron micrographs of lyophilized reaction mixtures revealed highly crystalline domains. Indeed, preliminary diffraction studies provided a partial structure of the TEMPO- $\mathrm{N}_{3}$ adduct consistent with the calculated CTC 1, but unambiguous structural characterization was hampered by low-quality data and confounding diffraction from crystalline impurities present in the reaction mixture. To prepare the CTC in a 
pure form, we devised an electrolysis experiment, in which a constant current of $5 \mathrm{~mA} / \mathrm{cm}^{3}$ (geometrical current density) was passed through a solution of TEMPO and $\mathrm{NaN}_{3}$ in $\mathrm{MeCN}$ and $\mathrm{H}_{2} \mathrm{O}$ using a carbon felt anode and a $\mathrm{Pt}$ cathode. The addition of $\mathrm{H}_{2} \mathrm{O}$ as a cosolvent rendered this experiment free of any exogenous electrolyte (e.g., $\mathrm{LiClO}_{4}$ ). By using aqueous $\mathrm{NaN}_{3}$ as the electrolyte, the subsequent purification and crystallization process was significantly simplified. The electrolysis mixture, presumably consisting of TEMPO-N $\mathrm{N}_{3} \mathrm{CTC} \mathbf{1}$ and $\mathrm{NaOH}$, was then subjected to a rapid workup procedure to remove $\mathrm{NaOH}$. Upon solvent evaporation, the resultant mixture of CTC 1 and its decomposition product TEMPO was further purified via vacuum sublimation. During the Kugelrohr fractional sublimation, we discovered that TEMPO and TEMPO- $\mathrm{N}_{3}$ sublime at markedly different temperatures $\left(19{ }^{\circ} \mathrm{C}\right.$ and $50{ }^{\circ} \mathrm{C}$, respectively, at a constant pressure of 200 mtorr). This difference allowed us to isolate dark, opaque, shock-sensitive crystals that were 30-100 microns in diameter, much too large and far too sensitive for milling to proper size for electron crystallography. Importantly, dissolution of this material in $\mathrm{MeCN}$ regenerated the distinct spectroscopic features $\left(\lambda_{\max }=380 \mathrm{~nm}\right)$ diagnostic of the TEMPO-N ${ }_{3}$ CTC (4). Warning: This solid is shock-sensitive and can rapidly decompose in a range of solvents and crystallography oils.

We then subjected these crystals to $\mathrm{Cu} \mathrm{K \alpha}$ X-ray diffraction and obtained a structural solution remarkeably consistent with the calculated structure of the TEMPO-N 3 CTC (1) (Fig. 2A, left). Synchrotron X-ray diffraction data was also collected from the same crystal, providing a higher-resolution structure and more complete reflection set (Fig. 2A right and 2B) (1.05 $\AA$ vs. $1.35 \AA$ ) in excellent agreement with our $\mathrm{Cu} \mathrm{K \alpha}$ solution (Fig. $2 \mathrm{C})$, albeit with a higher $R$-factor $(R$ $=0.105$ vs. 0.049 ). The X-ray data confirmed the structure predicted by quantum mechanical density functional theory (DFT) calculations (Fig. 2D, left), with the azide moiety positioned directly over the oxoammonium $\mathrm{N}=\mathrm{O}$ bond. This structure features a nonlinear azide with an unusual $\mathrm{N}-\mathrm{N}-\mathrm{N}$ bond angle of $170.0(6)^{\circ}\left(169.9^{\circ}\right.$ calculated $)$, a geometry distinct from canonically linear azidyl radicals and azide anions. Although slightly bent metal azides $\left(174-177^{\circ}\right)$ have been reported $(16,17)$, this structure is a unique example of azide nonlinearity engendered by a chargetransfer complex. In addition to this unique structural feature, two additional observations provide evidence of dynamic charge transfer and partial covalent bonding between $\mathrm{N}_{3}$ and TEMPO. First, the interatomic distances between the terminal nitrogen atoms on the azide and the atoms of the oxoammonium moiety $(2.337(6) \AA$ and $2.440(7) \AA)$ are contracted within the sum of the van der 
Waals radii, but substantially longer than a formal covalent bond. Interestingly, these distances are closely related to those found in transition states of alkyl and aryl azide cycloaddition reactions (2.1-2.5 $\AA)(18,19)$. Second, the aminoxyl N-O bond length of 1.247(4) $\AA$ of TEMPO-N 3 (1) lies in between the $\mathrm{N}-\mathrm{O}$ single bond $(1.28 \AA)$ in TEMPO and the $\mathrm{N}=\mathrm{O}$ double bond $(1.18 \AA)$ of $\mathrm{TEMPO}^{+}(20)$. These properties are characteristic of 4-center, 2-electron "pancake bonding" between two open-shell species $(21,22)$. The bond orders of the partially formed bonds between TEMPO and $\mathrm{N}_{3}$ motifs are 0.24 (for $\mathrm{NN}$ ) and 0.14 (for $\mathrm{NO}$ ), which are also characteristic of such 4-center, 2-electron bonding. Dissociation to the two component radicals is computed to require only $6-10 \mathrm{kcal} / \mathrm{mol}$, accounting for the ability of this complex to generate radicals to facilitate the catalytic cycle described previously $(4,5)$. Although crystal structures of $\left(\mathrm{TEMPO}^{+} \mathrm{X}^{-}\right)$-type CTCs have been reported by mixing TEMPO with a strong oxidant (e.g., $\mathrm{Br}_{2}$ or quinone derivatives), these structures display an $\mathrm{N}-\mathrm{O}$ bond distance of $c a .1 .19 \AA$ that is characteristic of TEMPO ${ }^{+}$with no apparent structural distortions or molecular interactions between the oxoammonium ion and the counter anion $\left(\mathrm{X}^{-}\right)$beyond ion pairing $(23,24)$. These features indicate near complete charge transfer from TEMPO to the oxidant and thus are distinct from the TEMPO-N $\mathrm{N}_{3} \mathrm{CTC}$ that we report herein.

Due to the instability of the CTC in solution, analyzing the electronic structure of the complex by electron spin resonance spectroscopy proved to be experimentally difficult. DFT calculations predicted a closed-shell species rather than a triplet radical pair. The HOMO of the complex resembles one of the degenerate azide HOMOs and resides exclusively on the azide, while the orthogonal orbital is stabilized by mixing with the TEMPO SOMO (which is the LUMO of TEMPO ${ }^{+}$(Fig. 2D, right and Fig. S7). The stunning resemblance between the crystal and DFTpredicted structures indicates that the CTC is a closed-shell species that arises from interaction between the SOMOs of the TEMPO and azidyl radicals (25). The DFT-predicted charge distribution revealed a significant degree of charge redistribution, with 0.62 electron transfer from $\mathrm{N}_{3}{ }^{-}$to $\mathrm{TEMPO}^{+}$, or 0.38 electron transfer from TEMPO to $\mathrm{N}_{3}{ }^{\cdot}$, depending on the reference starting point of CTC formation. Thus, despite being a closed-shell complex, CTC 1 lies closer in electronic structure to an interacting radical pair than an ion pair. 

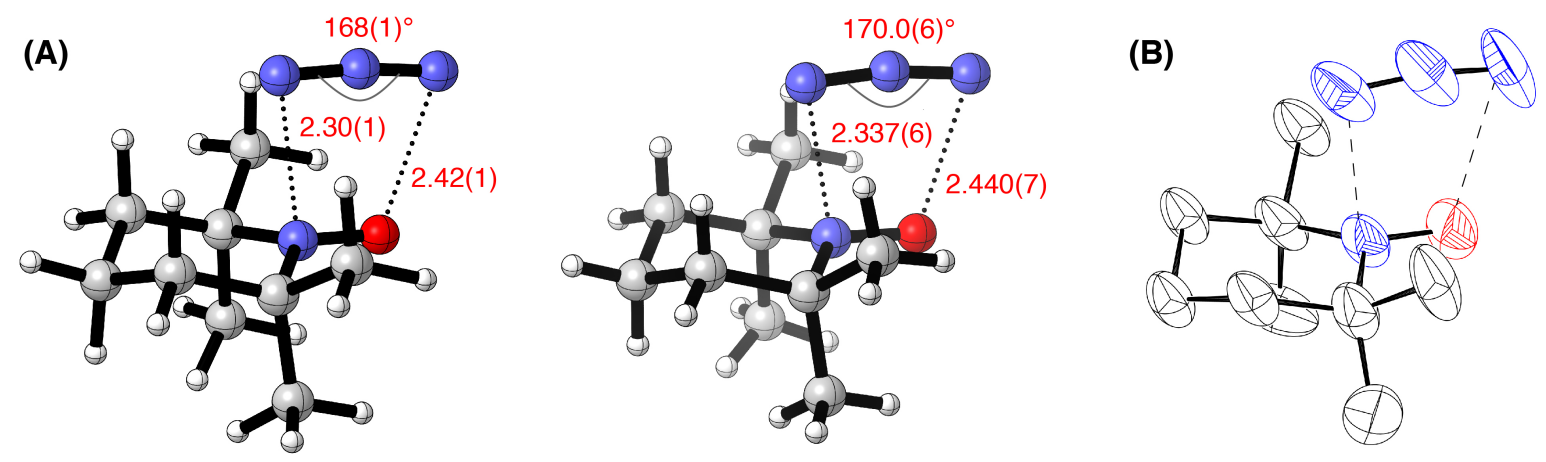

(C)
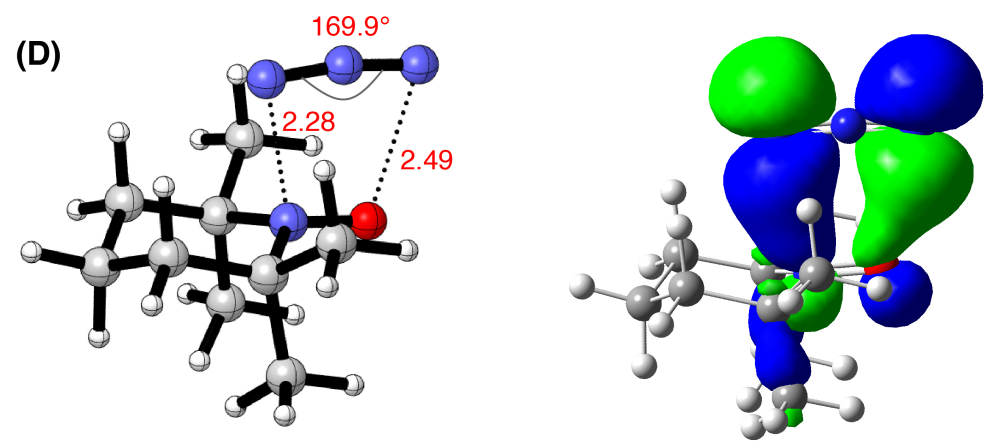

Fig. 2. Experimental and computed structures of CTC 1. (A) X-ray crystal structure of 1, Cu Ka (1.5418 $\AA)$ (left) and synchrotron radiation $(0.7749 \AA)$ (right); (B) ORTEP diagram of the synchrotron X-ray structure of 1 with ellipsoids shown at the $30 \%$ probability level, with $\mathrm{H}$ atoms omitted for clarity; (C) Overlay of structures obtained from $\mathrm{Cu} \mathrm{K \alpha}$ (green) and synchrotron radiation (blue), root-mean-square deviation $(\mathrm{RMSD})=0.028 \AA$ (for all non-hydrogen atoms) or $0.015 \AA$ for the $\mathrm{N}=\mathrm{O}-\mathrm{N}_{3}$ motif. (D) DFT predicted structure at B3LYP-D3/6-311++G(d,p) level of density functional theory. For a previously reported DFT structure at a lower level of theory, see ref. 4. (E) Electronic structure (HOMO-1) of 1 calculated at B3LYP-D3/6-311++G(d,p) level of theory. See Supplementary Material for other key molecular orbitials.

In conclusion, we report the X-ray crystal structure of a TEMPO-N 3 CTC (1). Our highresolution structure, reminiscent of a [3+2] cycloaddition transition state, features an unusual azide moiety that is significantly distorted from linearity to facilitate the 4-center, 2-electron bonding in CTC. The experimentally determined structure is remarkably consistent with the calculated structure, complete with partial bonding indicative of weak covalent interactions stabilizing the complex, and easily dissociated to a reactive radical pair. The definitive structural elucidation of the CTC lends further support to our proposed mechanism of the electrochemical alkene azidation reactions that it mediates.

\section{References:}

1. J. K. Kochi, Chemical Activation by Electron Transfer in Charge-Transfer Complexes. 
Formation and Reactions of Transient Ion Radical Pairs. Acta Chem. Scand. 44, 409-432 (1990).

2. Ł. Woźniak, J. J. Murphy, P. Melchiorre, Photo-organocatalytic Enantioselective Perfluoroalkylation of $\beta$-Ketoesters. J. Am. Chem. Soc. 137, 5678-5681 (2015).

3. E. Arceo, A. Bahamonde, G. Bergonzini, P. Melchiorre, Enantioselective Direct $\alpha-$ Alkylation of Cyclic Ketones by Means of Photo-organocatalysis. Chem. Sci. 5, 2438-2442 (2014).

4. J. C. Siu et al., Electrochemical Azidooxygenation of Alkenes Mediated by a TEMPO-N 3 Charge-Transfer Complex. J. Am. Chem. Soc. 140, 12511-12520 (2018).

5. J. C. Siu, J. B. Parry, S. Lin, Aminoxyl-Catalyzed Electrochemical Diazidation of Alkenes Mediated by a Metastable Charge-Transfer Complex. J. Am. Chem. Soc. 141, 2825-2831 (2019).

6. D. Josephy, T. Eling, R. Mason, The Horseradish Peroxidase-catalyzed Oxidation of 3,5,3',5'-Tetramethylbenzidine. J. Biol. Chem. 257, 3669-3675 (1982).

7. K. F. Biegasiewicz et al., Photoexcitation of flavoenzymes enables a stereoselective radical cyclization. Science 364, 1166-1169 (2019).

8. S. V. Rosokha, J. K. Kochi, Mechanism of Inner-sphere Electron Transfer via Chargetransfer (precursor) Complexes. Redox Energetics of Aromatic Donors with the Nitrosonium Acceptor. J. Am. Chem. Soc. 123, 8985-8999 (2001).

9. H. Kobayashi, J. Nakayama, The Crystal Structure of the Charge-transfer Complex of Dibenzotetrathiafulvalene-Tetracyanoquinodimethane, DBTTF-TCNQ. Bull. Chem. Soc. Jpn. 54, 2408-2411 (1981).

10. T. Murata et al., A Purely Organic Molecular Metal Based on a Hydrogen-Bonded Chargetransfer Complex: Crystal Structure and Electronic Properties of TTF-Imidazole- $p$ Chloranil. Angew. Chem., Int. Ed. 43, 6343-6346 (2004).

11. D. V. Konarev et al., Synthesis, Crystal Structure and Some Properties of Charge Transfer Complex of C60 with Asymmetric Donor TMDTDM-TTF. Synth. Met. 88, 85-87 (1997).

12. T. Horibe, S. Ohmura, K. Ishihara, Structure and Reactivity of Aromatic Radical Cations Generated by $\mathrm{FeCl}_{3}$. J. Am. Chem. Soc. 141, 1877-1881 (2019).

13. A. Samuni et al., Kinetics and Mechanism of Hydroxyl Radical and OH-Adduct Radical Reactions with Nitroxides and with Their Hydroxylamines. J. Am. Chem. Soc. 124, 87198724 (2002).

14. B. Zhang, A. Studer, Stereoselective Radical Azidooxygenation of Alkenes. Org. Lett. 15, 4548-4551 (2013).

15. C. G. Jones et al., The CryoEM Method MicroED as a Powerful Tool for Small Molecule Structure Determination. ACS Cent. Sci. 4, 1587-1592 (2018).

16. S. Öz et al., Two Non-Linear Azide Containing Heteronuclear Complexes: Crystal Structure and Thermal Decomposition. J. Therm. Anal. Calorim. 99, 363-368 (2010).

17. W. K. Seok, T. M. Klapötke, Inorganic and Transition Metal Azides. Bull. Korean Chem. Soc. 31, 781-788 (2010).

18. F. Schoenebeck, D. H. Ess, G. O. Jones, K. N. Houk, Reactivity and Regioselectivity in 1,3Dipolar Cycloadditions of Azides to Strained Alkynes and Alkenes: A Computational Study. J. Am. Chem. Soc. 131, 8121-8133 (2009).

19. S. Xie, S. A. Lopez, O. Ramstrom, M. Yan, K. N. Houk, 1,3-Dipolar Cycloaddition Reactivities of Perfluorinated Aryl Azides with Enamines and Strained Dipolarophiles. $J$. Am. Chem. Soc., 137, 2958-2966 (2015). 
20. Y. Yonekuta, K. Oyaizu, H. Nishide, Structural Implication of Oxoammonium Cations for Reversible Organic One-electron Redox Reaction to Nitroxide Radicals. Chem. Lett. 36, 866 (2007).

21. J. S. Miller, J. J. Novoa, Four-Center Carbon-Carbon Bonding. Acc. Chem. Res. 40, 189196, (2007).

22. Z.-h. Cui, H. Lischka, T. Mueller, F. Plasser, M. Kertesz, Study of the Diradicaloid Character in a Prototypical Pancake-Bonded Dimer: The Stacked Tetracyanoethylene (TCNE) Anion Dimer and the Neutral $\mathrm{K}_{2} \mathrm{TCNE}_{2}$ Complex. ChemPhysChem 15, 165-176, (2014).

23. Nakatsuji, S. et al. CT Complexes Based on TEMPO Radicals. J. Mater. Chem. 9, $1747-$ 1754 (1999).

24. Percino, M. J. et al. 2,2,6,6-Tetramethyl-1-oxopiperidinetribromide and Two Forms of 1Hydroxy-2,2,6,6-tetramethylpiperidinium Bromide Salt: Syntheses, Crystal Structures and Theoretical Calculations. J. Mol. Struct. 1103, 254-264 (2016).

25. T. Stuyver et al., Do Diradicals Behave Like Radicals? Chem. Rev. 119, 11291-11351 (2019).

Acknowledgments: The authors thank Prof. Sarah Tolbert (UCLA), Prof. Kyle Lancaster, Dr. Samantha MacMillan, Prof. Roald Hoffmann (Cornell), and Dr. Bo Chen (Donostia International Physics Center) for helpful discussions, Dr. Michael R. Sawaya and Michael J. Collazo (UCLA) for assistance with data collection and processing, and Dr. Michael D. Bridges (UCLA) for assistance with ESR spectroscopy. Financial support was provided by National Science Foundation (CHE-1751839 to S.L.; CHE-1764328 to K.N.H.) and Bristol Myers Squibb (Unrestricted Grant in Organic Chemistry; H.M.N). A.S. thanks the National Science Foundation for a Graduate Research Fellowship (DGE-1650604). This work incorporates data collected at the Northeastern Collaborative Access Team (NE-CAT) beamlines at Argonne National Laboratory. NE-CAT is funded by the NIH-NIGMS (P30 GM124165). The Pilatus 6M detector on the 24-ID$\mathrm{C}$ beam line is funded by NIH-ORIP HEI (S10 RR029205). This research used resources at the Advanced Photon Source, a U.S. Department of Energy Office of Science User Facility operated by Argonne National Laboratory under Contract No. DE-AC02-06CH11357. The UCLA-DOE Institute's X-ray Crystallography Core Facility is supported by the U.S. Department of Energy (DE-FC02-02ER63421). X-ray crystallographic data for CTC 1 has been deposited to Cambridge Crystallographic Data Center (CCDC 1994307, 1994308). All data is available in the main text or the supplementary materials. 